\title{
Characterizing Non-nesting for the Neyman-Pearson Family of Tests
}

\author{
Rahul Bhattacharya \\ Department of Statistics, University of Calcutta, \\ 35 Ballygunge Circular Road, Kolkata-700019, India \\ rahul_bhattya@yahoo.com
}

Received 24 April 2014

Accepted 2 July 2016

\begin{abstract}
For testing a simple null hypothesis against a simple alternative using Neyman-Pearson theory, examples of most powerful non-randomized critical regions are constructed, which are overlapping for varying sizes. A likelihood ratio based criterion, characterizing such critical regions, is also provided. A simple method, in addition, is suggested to construct the class of distributions providing overlapping critical regions for unequal sizes. These examples, in fact, counterexamples are important in explaining the fact that power of an optimum test may not increase with an increase in size.
\end{abstract}

Keywords: Nested critical region; Most Powerful test

2000 Mathematics Subject Classification: 62F03

\section{Introduction}

Most powerful critical regions for testing a simple null hypothesis against a simple alternative are, in general, nested, that is, the smaller the size, the smaller is the rejection region. Moreover, nested critical regions form the basis of the definition of $p$-value (see, for example, [1] ,page 63). However, the possibility of a reverse scenario, that is, non-nested critical regions for varying sizes, can not be ruled out. One such exception, based on discrete distributions, appeared in [2], which gives disjoint (i.e. non-nested) critical regions for most powerful non-randomized tests of unequal sizes. But they did not provide any criteria to characterize non-nesting. The primary objective of the current work is to develop a template to characterize non-nesting. We start with some exceptions for both discrete and continuous distributions and develop the criteria in an inductive way. All these can be found in Sections 2 and 3. In addition, starting from the likelihood ratio, a general method for deriving a number of overlapping(i.e. non-nested) critical regions is also provided in Section 3. Finally, Section 4 concludes with some related discussion. 


\section{Few exceptions of nesting}

\section{Consider testing}

$$
H: X \sim p_{0}(x) \text { against } K: X \sim p_{1}(x)
$$

where $p_{1}$ and $p_{0}$ are completely specified. Then, the Neyman-Pearson theory gives the most powerful size $\alpha$ test $\phi_{\alpha}=\phi_{\alpha}(x)$, defined as below:

$$
\begin{aligned}
\phi_{\alpha} & =1 \text { if } r(x)>k \\
& =\gamma \text { if } r(x)=k \\
& =0 \text { if } r(x)<k,
\end{aligned}
$$

where $k$ and $\gamma \in(0,1)$ satisfy $E_{H} \phi_{\alpha}=\alpha$ and $r(x)=\frac{p_{1}(x)}{p_{0}(x)}$ is the likelihood ratio(LR). If $\phi_{\alpha}$ takes only the values 0 and 1 , the test is non-randomized and randomized otherwise. If the most powerful size $\alpha$ critical region is denoted by $S_{\alpha}$, then naturally $S_{\alpha}=\left\{x: \phi_{\alpha}(x)=1\right\}$. Now most powerful critical regions $S_{\alpha}$ and $S_{\alpha^{\prime}}$ are said to be nested if $S_{\alpha} \subset S_{\alpha^{\prime}}$ for $\alpha<\alpha^{\prime}$ (see, for example, [1], page 63).However, the counterexample of Romano and Siegel([2],page 251), in this context, demonstrates that violation to the phenomena of nesting is also possible. In particular, considering specific probability mass functions $p_{0}$ and $p_{1}$, they illustrated non-nesting through disjoint critical regions $S_{\alpha}$ and $S_{\alpha^{\prime}}$, where $\alpha<\alpha^{\prime}$. We start with the counterexample of Romano and Siegel[2] and extend suitably to get most powerful critical regions $S_{\alpha}$ and $S_{\alpha^{\prime}}$, sharing some common points even for continuous distributions, whenever $\alpha<\alpha^{\prime}$ is satisfied.

Example 1 (Romano and Siegel[2]). In the context of (2.1), Romano and Siegel[2] considered specific choices of $p_{1}$ and $p_{0}$ to contradict the usual nesting property. The choices together with the related likelihood ratio(LR), are given in Table 1 below:

Table 1. Counterexample of Romano and Siegel[2].

\begin{tabular}{llll}
\hline$x$ & 1 & 2 & 3 \\
\hline$p_{0}(x)$ & 0.85 & 0.10 & 0.05 \\
$p_{1}(x)$ & 0.70 & 0.20 & 0.10 \\
$r(x)$ & 0.823 & 2.00 & 2.00 \\
\hline
\end{tabular}

Thus corresponding to $\alpha=.05$ and $\alpha^{\prime}=.10$, we have unique non-randomized most powerful critical regions $S_{\alpha}=\{3\}$ and $S_{\alpha^{\prime}}=\{2\}$, respectively. Naturally, the critical regions are neither nested nor overlapping.

Then the following questions are immediate and of pedagogic importance:

(a) Do there exist critical regions with ordered sizes having some points in common? and

(b) Can we get non-nested critical regions for continuous distributions?

In the following examples(i.e. examples 2,3 and 4), we try to answer the questions posed above. 
Example 2. Now consider the following choices of $p_{1}$ and $p_{0}$ :

Table 2. Counterexample for discrete $X$ : Overlapping Support.

\begin{tabular}{llllll}
\hline$x$ & 1 & 2 & 3 & 4 & 5 \\
\hline$p_{0}(x)$ & 0.85 & 0.10 & 0.025 & 0.00 & 0.025 \\
$p_{1}(x)$ & 0.70 & 0.20 & 0.05 & 0.05 & 0.00 \\
$r(x)$ & 0.823 & 2.00 & 2.00 & $\infty$ & 0.00 \\
\hline
\end{tabular}

A simple manipulation of $\{x: r(x)>=<k\}$ with $k=2$ gives most powerful non-randomized critical regions $S_{\alpha}=\{3,4\}$ and $S_{\alpha^{\prime}}=\{2,4\}$ with respective sizes $\alpha=.025$ and $\alpha^{\prime}=.10$. Then the critical regions are non-nested but overlapping. However, $p_{1}$ and $p_{0}$ do not have a common support.

Example 3. If we consider the following choices of $p_{1}$ and $p_{0}$,

Table 3. Counterexample for discrete $X$ : Common Support.

\begin{tabular}{lllllll}
\hline$x$ & 0 & 1 & 2 & 3 & 4 & 5 \\
\hline$p_{0}(x)$ & 0.05 & 0.02 & 0.03 & 0.05 & 0.35 & 0.50 \\
$p_{1}(x)$ & 0.10 & 0.03 & 0.045 & 0.15 & 0.14 & 0.535 \\
$r(x)$ & 2.00 & 1.50 & 1.50 & 3.00 & 0.40 & 1.07 \\
\hline
\end{tabular}

then corresponding to $\alpha=.12$ and $\alpha^{\prime}=.13$ we have non-randomized most powerful critical regions $S_{\alpha}=\{0,1,3\}$ and $S_{\alpha^{\prime}}=\{0,2,3\}$, respectively. Clearly, $S_{\alpha}$ is not contained in $S_{\alpha}$ for $\alpha<\alpha^{\prime}$ and unlike the counterexample of Romano and Siegel[2], $S_{\alpha} \cap S_{\alpha^{\prime}}$ is non empty.

Example 4. Next consider the following choices, where $p_{1}$ and $p_{0}$ are both density functions.

Table 4. Counterexample for continuous $X$.

\begin{tabular}{lrccc}
\hline$x$ & $(0,1)$ & $(1,2)$ & $(2,3)$ & $(3,4)$ \\
\hline$p_{0}(x)$ & $\frac{\sqrt{x}}{2}$ & $\frac{1}{3}$ & $\frac{1}{6}$ & $\frac{x}{21}$ \\
$p_{1}(x)$ & $\frac{x}{4}$ & $\frac{1}{2}$ & $\frac{1}{4}$ & $\frac{x}{28}$ \\
$r(x)$ & $\frac{\sqrt{x}}{2}$ & $\frac{3}{2}$ & $\frac{3}{2}$ & $\frac{3}{4}$ \\
\hline
\end{tabular}

For $k=\frac{3}{2}$, a routine consideration of $\{x: r(x)>=<k\}$ yields most powerful non-randomized critical regions $S_{\alpha}=(2,3)$ and $S_{\alpha^{\prime}}=\left(\frac{3}{2}, \frac{5}{2}\right)$ with sizes $\alpha=\frac{1}{6}$ and $\alpha^{\prime}=\frac{1}{4}$, respectively. Clearly $S_{\alpha}$ is not a subset of $S_{\alpha^{\prime}}$ and in fact $S_{\alpha} \cap S_{\alpha^{\prime}}=\left(2, \frac{5}{2}\right)$ is non empty. This particular example gives an extension of the counterexample of Romano and Siegel[2] for continuous distributions.

\section{Characterizing non-nesting}

The examples discussed so far though artificially constructed but are similar with respect to the behaviour of the likelihood ratio $r(x)$. In fact, in all these examples, LR is a constant for at least two support points. Consequently one can presume non-nesting under such a structure of LR. The following result gives a formal justification to the phenomena observed so far. 
Result. For testing $H: X \sim p_{0}(x)$ against $K: X \sim p_{1}(x)$, if the likelihood ratio $r(x)=\frac{p_{1}(x)}{p_{0}(x)}$ is a constant for at least two support points then there exist non-randomized most powerful critical regions which are overlapped for ordered sizes.

Proof. We assume that there exists some finite $s$ such that the domain of $r(x)$ can be split into sets $A^{+}=\{x: r(x)>s\}, A^{=}=\{x: r(x)=s\}$ and $A^{-}=\{x: r(x)<s\}$, where either of $A^{+}$or $A^{-}$may be empty but $A^{=}$contains at least two distinct values of $x$ or an interval of the real line. Then a routine manipulation of $\{x: r(x)>=<k\}$ for $k=s$ provides the most powerful test $\phi^{0}$, defined by,

$$
\begin{aligned}
\phi^{0} & =1 \text { if } x \in A^{+} \\
& =0 \text { if } x \in A^{-},
\end{aligned}
$$

where $\phi^{0}$ can take any value in $[0,1]$ for $x \in A^{=}$. Assigning $\phi^{0}=0$ or 1 to different subsets of $A^{=}$, one can get a number of non-randomized most powerful tests. Specifically, if we take $\phi^{0}=1$ or 0 as $x \in B \subset A^{=}$or $x \in A^{=}-B$, we get the most powerful non-randomized test

$$
\begin{aligned}
\phi & =1 \text { if } x \in A^{+} \cup B \\
& =0 \text { otherwise }
\end{aligned}
$$

with size $\alpha=E_{H} \phi=P_{H}\left(X \in A^{+}\right)+P_{H}(X \in B)$. Again the choice $\phi^{0}=1$ or 0 according as $x \in B^{\prime} \subset$ $A^{=}$or $x \in A^{=}-B^{\prime}$ for some $B^{\prime}$, leads to the most powerful non-randomized test

$$
\begin{aligned}
\phi^{\prime} & =1 \text { if } x \in A^{+} \cup B^{\prime} \\
& =0 \text { otherwise }
\end{aligned}
$$

with size $\alpha^{\prime}=E_{H} \phi^{\prime}=P_{H}\left(X \in A^{+}\right)+P_{H}\left(X \in B^{\prime}\right)$. If $B$ and $B^{\prime}$ are chosen such that $B \subset B^{\prime}$, then not only $\alpha<\alpha^{\prime}$ is satisfied but the corresponding critical regions also share some common points(i.e. $B$ in this case). This proves the claim of the existence of non nested critical regions under ordered sizes.

Remarks: The above result not only provides the condition of existence of a non nested critical region under ordered sizes but also gives a possible way of getting a number of choices of $p_{0}$ and $p_{1}$ leading to non nesting through an examination of $r(x)$. For example, suppose $X$ is distributed over $\{0,1,2,3\}$ keeping $r(x)=\frac{s}{2} I_{\{0\}}(x)+s I_{\{0,1\}}(x)+2 s I_{\{3\}}(x)$ for some $s$, where $I_{A}($.$) is the usual indi-$ cator function. Then a routine manipulation of the set $\{x: r(x)>=<s\}$ gives most powerful nonrandomized overlapping critical regions $S_{\alpha}=\{1,3\}$ and $S_{\alpha^{\prime}}=\{2,3\}$. Now, if we choose $p_{0}(x)$ satisfying $P_{H}(X=1)<P_{H}(X=2)$, then we have $\alpha<\alpha^{\prime}$, in addition to non-nesting. Although an infinite number of choices are possible, we consider the following choice $p_{0}(x)=\frac{1}{6} I_{\{0,1\}}(x)+\frac{1}{3} I_{\{2,3\}}(x)$ for illustration. Since $r(x)=\frac{p_{1}(x)}{p_{0}(x)}$, we can get $p_{1}(x)$ and the use of the identity $\sum_{x=0}^{3} p_{1}(x)=1$ specifies $p_{1}(x)$ explicitly. Therefore, starting from a particular nature of $r(x)$, a number of choices of $p_{1}$ and $p_{0}$ providing non nested most powerful non-randomized critical regions for ordered sizes can be derived. It is worth mentioning at this point that we adopted the same mechanism to construct examples 2, 3 and 4 . 


\section{Concluding Remarks}

Starting from the review of an well known counterexample, a general criteria to characterize nonnesting under ordered sizes even for continuous distributions is developed. Most importantly, a simple method is suggested to derive reasonable choices of $p_{0}$ and $p_{1}$ from non-nested critical regions. The entire work is based on the critical regions corresponding to most powerful non-randomized tests of certain sizes. But tests can be randomized, and in such a case nesting is defined in terms of the test functions (see, for example, [1], page 64). It is interesting to note that such a definition may lead to ambiguity in case of non-randomized tests(e.g. Example 3). However, the basic aim of the current work is to develop an insight on nesting of critical regions under the Neyman-Pearson paradigm of hypothesis testing, not to explain the role of randomization and hence we omit the details associated with randomization.

\section{Acknowledgments}

The author would like to thank the anonymous reviewers, the editor and the associate editor for their concerns and insightful comments.

\section{References}

[1] E. L. Lehmann and J. P. Romano, Testing Statistical Hypotheses, Third Edition, (Springer,New York, 2005).

[2] J. P. Romano and A.F. Siegel, Counterexamples in Probability And Statistics, (Wadsworth, Belmont,1986). 